\title{
Minimizing the surface effect of PDMS-glass microchip on polymerase chain reaction by dynamic polymer passivation
}

\author{
Yong-Mei Xia, ${ }^{1,2 *}$ Zhi-Shan Hua, $^{2}$ Onnop Srivannavit, ${ }^{2}$ Ayse Bilge Ozel ${ }^{2}$ and \\ Erdogan Gulari2** \\ ${ }^{1}$ School of Chemical and Material Engineering, Southern Yangtze University, 170\# Huihe Road, Wuxi, Jiangsu 214036, China \\ ${ }^{2}$ Department of Chemical Engineering, University of Michigan, 3085 H.H. Dow, Ann Arbor, MI 48109, USA
}

\begin{abstract}
Polydimethyl siloxane (PDMS)-glass microchip has a very strong surface effect on polymerase chain reaction (PCR), leading to a very poor PCR yield. In the work reported here, practical dynamic passivation of surfaces of PDMS-glass microchip using polyethylene glycol (PEG) or polyvinylpyrrolidone (PVP) was achieved using a conventional thermocycler. The passivation procedure was cost-effective and easy to conduct. The effects of polymer molecular weight and polymer concentration on tube PCR efficiency were investigated primarily to prescreen out suitable polymers and polymer concentrations in the PCR mixture. The result from tube PCR indicated that both PEG and PVP could affect the performance of Taq polymerase. A final concentration of $0.025 \%$ (w/v) or $0.4 \%$ (w/v) polymer in the PCR mixture can enhance the tube PCR, while $1 \%$ (w/v) polymer was found to inhibit the reaction. PEG was more effective in tube PCR, although PVP performed better in chip PCR. Instead of employing the polymer directly in the PCR mixture, i.e. the conventional in situ passivation approach, another approach of dynamic passivation by pre-injecting polymers into the microchip achieved better performance. The efficiency of pre-passivation was found to follow the order: PVP10000 > PVP55000, PEG8000> PEG10000>PEG400. After pre-passivation with PVP10000, PVP55000 and PEG8000, the PCR efficiency can recover to $93 \%$, 86\% and $83 \%$, respectively, of that obtained from tube PCR.

(C) 2006 Society of Chemical Industry
\end{abstract}

Keywords: dynamic passivation; PDMS; PCR; microchip; PVP; PEG

\section{INTRODUCTION}

Polydimethyl siloxane (PDMS) has become an attractive structural material for microreactors because of its low cost and ease of processing. A typical technique to fabricate PDMS/glass microchip is to bond a patterned PDMS layer onto a glass slide to form closed chambers or microfluidic channels, which can be used for a polymerase chain reaction (PCR) or other applications. ${ }^{1-4}$ However, both PDMS and glass as microchip substrates are less than ideal for PCR because of the proclivity of Taq polymerase to be adsorbed onto the chip surface.

Surface passivation is essential for most kinds of PCR microchip made from different materials. A commonly used surface passivation protocol is static passivation, which is achieved by bovine serum albumin (BSA) adsorption, oxidation, silanization, or coating the polymer covalently onto the surface. ${ }^{5,6}$ Another protocol, dynamic passivation, is more convenient to use because it does not require additional steps to treat the surface. ${ }^{7-9}$
Many studies have been reported concerning the passivation of silicon-glass chip surfaces to prevent the adsorption of polymerase. However, few studies have reported on the passivation of PDMS-glass microchips. The documented passivation of PDMS-glass chip is based on a static approach, coating with BSA, or covalently coating a chemical polymer such as 2-methacryloyloxyethyl phosphorylcholine onto the PDMS surface followed by washing and curing the surface. ${ }^{10}$ These static coating methods are time-consuming, and there is a risk that the coatings will partially lift off the surface during the high temperature reaction.

Dynamic passivation of PDMS-glass PCR reactors has not been reported. In this study, we dynamically passivated PDMS-glass PCR microchip using polyethylene glycol (PEG) or polyvinylpyrrolidone (PVP). The effects of polymer molecular weight and polymer concentration in the PCR mixture on the PCR efficiency were investigated, primarily in tube PCR. Dynamic passivation was carried out using a pre-passivation approach and an in situ passivation

* Correspondence to: Yong-Mei Xia, School of Chemical and Material Engineering, Southern Yangtze University, Wuxi, Jiangsu 214036, China

E-mail: ymxia@126.com

Erdogan Gulari, Department of Chemical Engineering, University of Michigan, MI 48109, USA

E-mail: gulari@umich.edu

Contract/grant sponsor: NIH, USA; Michigan Life Sciences Corridor, Michigan; Program for New Century Excellent Talents in University by Ministry of Education, China

(Received 24 February 2006; revised version received 23 May 2006; accepted 25 May 2006)

Published online 24 November 2006; DOI: 10.1002/jctb.1631 
approach, and the performances of the two methods were compared. The possible role of polymers in PCR is proposed.

\section{EXPERIMENTAL}

\section{Reagents and apparatus}

PEG 400, PEG8000, PEG10000, PVP10000 and PVP55000 were purchased from Sigma (St. Louis, MO, USA) and used without further purification. Photoresist PR 1813 and developer solution were obtained from Hoechst Celanese (Corpus Christi, TX, USA). PDMS kit (Sylgard 184) including prepolymer and its curing agent was obtained from Dow Corning (Midland, MI, USA).

Resist stripper (Model PRS 2000) was obtained from JT Baker Inc. (Philippsburg, NJ, USA). The March Etcher (Model PX-1000) used was from March Instrument Co., California, USA. The PCR thermal cycler was from Thermo Hybaid PCR Express (Ashford, UK). The UV transilluminator (Model 88A) used was from Fisher Biotech (Fair Lawn, NJ, USA).

\section{PDMS-glass microchip fabrication (Fig. 1)}

A master silicon wafer with the desired microstructure was fabricated first, beginning by spin-coating a layer of the photoresist at $4000 \mathrm{rpm}$ for $30 \mathrm{~s}$ on a silicon wafer. The wafer was soft-baked at $90^{\circ} \mathrm{C}$ in an oven for $30 \mathrm{~min}$ and then exposed to UV radiation $\left(404.7 \mathrm{~nm}, 10 \mathrm{~mJ} \mathrm{~cm}^{-2}, 20 \mathrm{~s}\right)$ to define the desired microstructure. After removal of the activated photoresist with developer solution for $60 \mathrm{~s}$, the wafer was hard-baked at $110^{\circ} \mathrm{C}$ for $30 \mathrm{~min}$. The microstructure pattern was transferred to the silicon wafer by deep reactive ion etching. The photoresist was then removed with a resist stripper. To fabricate the PDMS microchip, PDMS prepolymer and its curing agent in the ratio 10:1 were well mixed and then degassed for $30 \mathrm{~min}$. The mixture was poured into the master and cured at $65^{\circ} \mathrm{C}$ for $1 \mathrm{~h}$. After curing, the PDMS replica was peeled off the master wafer. The PDMS replica and glass slide were treated by oxygen plasma in the March Etcher for $30 \mathrm{~s}$. With this treatment, glass and PDMS were bonded when brought in contact. Each PDMS-glass microchip consists of 12 reaction chambers. For each chamber, the ratio of surface area/volume is $21.6 \mathrm{~mm}^{2} \mu \mathrm{L}^{-1}$, about 14.4 times that of a MicroAmp reaction tube.

\section{Dynamic passivation on the PDMS-glass microchip}

The dynamic passivation of microchip PCR with PVP and PEG polymers was conducted using two different approaches: pre-passivation and in situ passivation, respectively.

For pre-passivation, 10 of the 12 chambers in the microchip were fully filled with polymer solution $(10 \%$ $(w / v)$ in water), of which every two chambers were pipetted with the same polymer solution; the remaining two chambers were filled with nuclease-free water

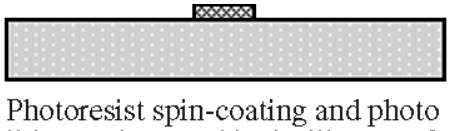

lithography on a blank silicon wafer

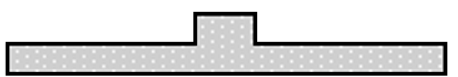

Pattern transfer onto silicon master wafer using deep reactive ion etching

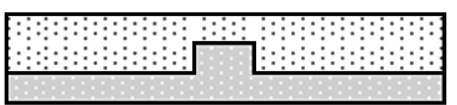

Pour PDMS prepolymer onto silane treated silicon master, followed by thermal curing of PDMS
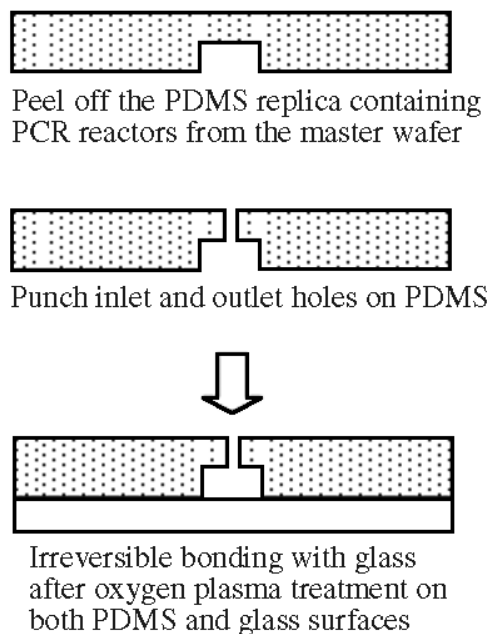

Silicon

Photoresist PDMS Glass

Figure 1. Fabrication of PDMS-glass chip (demonstration of a single chamber).

as a control. After $30 \mathrm{~min}$, the liquid was blown from the chambers by airflow $(1 \mathrm{~kg})$ for $30 \mathrm{~s}$. Each chamber was pipetted $5 \mu \mathrm{L}$ of PCR mixture. No polymer solution was added directly into the PCR mixture in this case. The microchips were then sealed with epoxy gel (Devcon, Danvers, MA, USA) and placed on the plate block in the thermal cycler for PCR amplification.

For in situ passivation, the reaction chambers were first washed using nuclease-free water, and then air-dried. The polymer solution $(10 \%(\mathrm{w} / \mathrm{v})$ in water) was added into the PCR mixture with a final polymer concentration of $0.4 \%(\mathrm{w} / \mathrm{v})$. PCR mixtures containing different polymers were injected into individual reaction chambers. The remaining procedures were the same as described for the 'prepassivation' samples.

\section{PCR using E. Coli genomic DNA as template}

PCR reactions using E. Coli genomic DNA as template were conducted in a GeneAmp reaction tube and PDMS-glass microchip using several approaches.

To investigate the polymer concentration and molecular weight dependence of PCR, amplifications 
were carried out in GeneAmp reaction tubes in the presence of polymers with final concentrations (w/v) of $0 \%, 0.025 \%, 0.4 \%$ and $1 \%$ in PCR mixtures. Two different approaches were compared: directly adding the polymer into the PCR mixture containing polymerase, or mixing the polymer with polymerase prior to adding it into the PCR mixture.

To test the dynamic passivation effect of polymer on chip PCR, the chips were treated and filled with PCR mixtures as described earlier.

The PCR mixture contained $200 \mu \mathrm{mol} \mathrm{L}^{-1} \mathrm{dNTP}$, $2.5 \mathrm{mmol} \mathrm{L}^{-1} \mathrm{MgCl}_{2}, \quad 0.25 \mathrm{U}$ Taq polymerase (Promega), PCR Buffer $\left(50 \mathrm{mM} \mathrm{KCl}, 10 \mathrm{mmol} \mathrm{L}^{-1}\right.$ Tris- $\mathrm{HCl}$, pH 9.0 (at $25^{\circ} \mathrm{C}$ ), $0.1 \%$ Triton $\mathrm{X}$ 100), $1 \mu \mathrm{mol} \mathrm{L}^{-1}$ each primer, and $100 \mathrm{ng} E$. Coli genomic DNA (Extracted from E. Coli O157:H7 strain). Primers for E. Coli dihydrofolate reductase (DHFR) generating a 601-bp PCR target were forward primer 5'-GATTACAAACGTTTGTAATCCCATTGTAATGCGGCGAGTCC-3' and reverse primer 5'-AACTAATTAGAATTCTAATTATGCAATAGCTGTGAGAGTTC-

TGC-3'. For both tube PCR and chip PCR, the amplification procedure was as follows: $2 \mathrm{~min}$ at $94^{\circ} \mathrm{C}$, followed by 35 cycles of $94^{\circ} \mathrm{C}$ for $30 \mathrm{~s}$, $55^{\circ} \mathrm{C}$ for $30 \mathrm{~s}, 72^{\circ} \mathrm{C}$ for $40 \mathrm{~s}$, and finally heated at $72^{\circ} \mathrm{C}$ for $5 \mathrm{~min}$. All PCR amplifications were run in parallel. All experiments were made at least in triplicate. Tube PCR running under the same conditions but without the addition of polymer was included in every experiment as a control.

The amplified products were detected using agarose gel electrophoresis and quantified with image analysis software (Image J Software $\mathrm{v} 1.32 \mathrm{j}$, $\mathrm{NIH}$, http://rsb.info.nih.gov/ij/) after the gel was photographed with the UV transilluminator. The brightness of the PCR product band on the gel was used to indicate the tube PCR efficiency, based on comparison with that of the control. Since chip PCR without passivation produces no amplification product, the passivation efficiency of the polymer was defined as the ratio of brightness of the PCR product band obtained from the PCR running in the presence of the polymer to that of control tube PCR running in the absence of the polymer.

\section{RESULTS AND DISCUSSION}

PEG is a well-known biocompatible polymer, and PVP has been recognized as a PCR enhancer. ${ }^{11}$ PEG has also been used as a liquid substrate to immobilize enzyme to improve the enzyme's activity and stability. ${ }^{12}$ Moreover, PEG and PVP are known to form loose and weak complexes with proteins; this may improve the hydrophilicity of the protein, and enhance the enzyme's physical and thermal stability. Thus the polymers may influence the properties of the polymerase depending on their molecular weight, structure and concentrations. All of these suggest the possibility that the interaction between polymerase and polymers may also have a potential benefit on PCR amplification.

To reveal the effect of the polymers on PCR and find the most effective polymer for passivation on PDMS-glass chip, PEG and PVP having different molecular weights and concentrations were tested in tube PCR first. Their performance in PCR reactions conducted in GeneAmp reaction tubes was investigated. Subsequently, dynamic passivation on PDMS-glass chips was studied.

\section{PCR in GeneAmp reaction tubes with polymers in the reaction mixture}

\section{Effect of polymer type, concentration and polymer} molecular weight

Figure 2 illustrates the effects of assayed polymers on PCR in GeneAmp reaction tubes; polymers were added into reaction mixtures already containing polymerase.

The results show that all assayed PEG samples were more effective than PVP samples in this experiment. All PEG samples tested provided PCR enhancement while PVP induced inhibition of PCR. Among the assayed polymers, PEG400 and PEG8000 performed best.

Regarding the effect of concentration, results indicate that $0.4 \%$ and $0.025 \%$ are suitable final polymer concentrations in PCR mixtures for both PEG and PVP. However, for concentrations of $1 \%$, PCR inhibition was observed in the presence of all polymers tested. This inhibition may be due to polymer-protein or polymer-DNA complex formation, which might prevent polymerase moving onto the DNA chain, and therefore inhibit PCR.

At lower concentrations $(0.025 \%, 0.4 \%)$, shorter PEG (PEG 400 and PEG8000) enhanced PCR better, which implies that improvement of the hydrophilic environment of polymerase may become

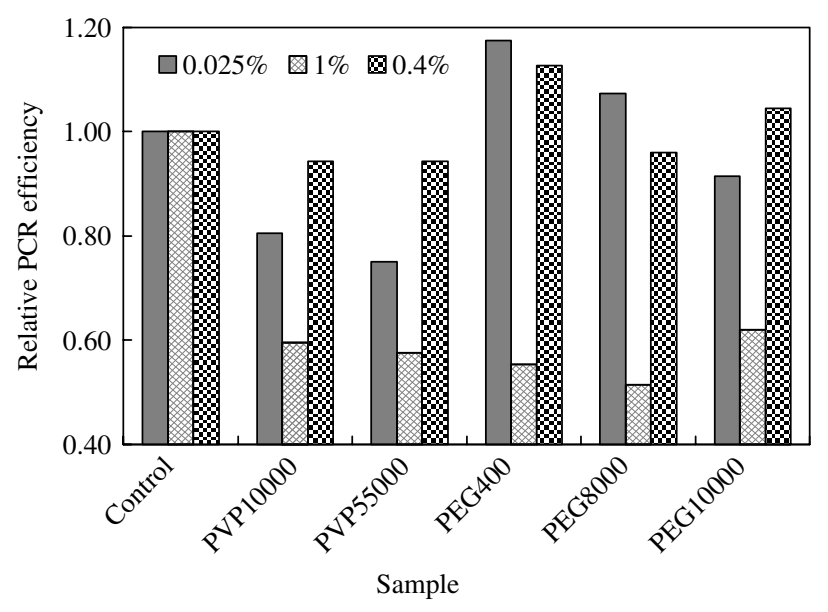

Figure 2. Effects of polymer concentration and molecular weight on tube PCR. Final polymer concentrations in PCR mixtures were $0.025 \%, 0.4 \%$ and $1 \%(\mathrm{w} / \mathrm{v})$, respectively. Control: PCR mixture containing no polymer. 
dominant. On the other hand, with the same weight concentration, the number of repeating units (indicating possible binding sites) in all samples containing PEG were almost same, which suggests that the interaction between longer PEG and the enzyme is weaker than that between shorter PEG and the enzyme.

\section{Pretreatment of polymerase with PEG and PVP}

Both PEG and PVP are hydrophilic polymers, which can influence enzyme activity by affecting the water structure surrounding the protein molecules and therefore affecting protein formation. The interaction between PEG or PVP and Taq polymerase has not been reported, but interactions of PEG or PVP with some other proteins (such as BSA) have been well studied. ${ }^{13,14}$ Farruggia et al. found an equilibrium constant value of about $10^{2} \mathrm{~L} \mathrm{~mol}^{-1}$ for BSA in low molecular weight PEG complex formation, which suggests a very weak interaction. ${ }^{15}$ They also reported $^{16}$ that in the presence of PEG 600-8000, a significant decrease in protein relative surface hydrophobicity was observed, but this effect had no obvious relationship with molecular weight of PEG.

Most researchers believe that in dynamic passivation PEG or PVP passivates surfaces by competitive adsorption with proteins. But as discussed above, the result from Fig. 2 suggests possible interaction between the polymer and the enzyme.

To further study this interaction, we modified the tube PCR approach by pretreatment of polymerase with PEG and PVP; i.e. polymers were mixed with polymerase first and then added to the reaction mixtures to intentionally enhance the possible assembly of polymer-polymerase.

Figure 3 illustrates a comparison of the previous approach, in which the polymers were added to the PCR mixture containing polymerase (PVP10000a, PEG10000a), with this proposed pretreatment (PVP10000b and PEG10000b) for tube PCR.

One can see from Fig. 3 that at all tested concentrations, PVP10000b gave a higher PCR yield than PVP10000a, and similarly, PEG10000b gave higher yield than PEG10000a. This result indicates that both PEG and PVP can affect the properties of the Taq polymerase, which further substantiates that there was an interaction between the polymers and polymerase. In addition, PEG10000b gave a higher PCR yield than PVP10000b, agreeing with the previous observation from Fig. 2, showing that with the similar molecular weight, PEG is more efficient than PVP. This difference may be explained by the positive charge of PVP, which may form complexes with DNA or dNTP due to charge-charge interaction, and may therefore have a negative effect on PCR.

\section{Dynamic passivation on PDMS-glass PCR microchip using PEG or PVP}

PEG is well known for its extraordinary ability to prevent protein adsorption. Modifying surfaces with

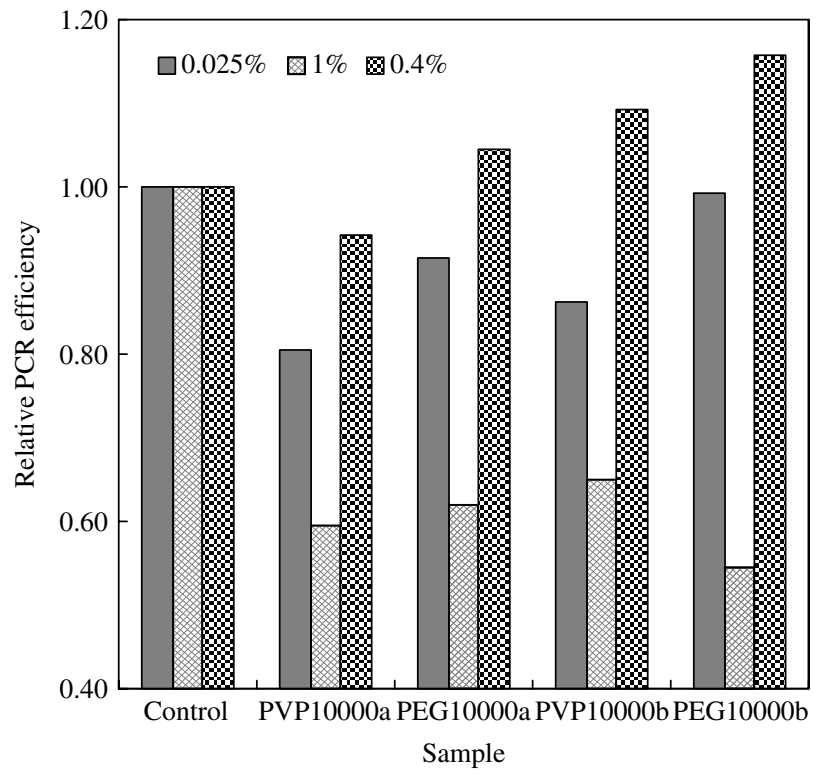

Figure 3. Effects of polymerase pretreatment with PVP10000 and PEG10000 on tube PCR. PVP10000a or PEG10000a: polymer was added into PCR mixture already containing polymerase. PVP10000b or PEG10000b: polymer was mixed with polymerase first and then added to the reaction mixture. Control: PCR mixture containing no polymer. Final polymer concentration in PCR mixtures was $0.025 \%$, $0.4 \%$ and $1 \%$, respectively.

PEG can improve blood compatibility and minimize protein adhesion. ${ }^{17,18}$ For passivation of silicon or glass surfaces, the effect of the molecular weight and density of PEG on passivation has been studied. ${ }^{14} \mathrm{On}$ PEG tethered asymmetric membranes, the amount of BSA adsorption depends on the molecular weight of PEG. The order of passivation efficiency is PEG400 > PEG600 > PEG1000 > PEG200.$^{5}$ However, similar studies have not been reported for dynamic passivation with PEG on glass or PDMS surfaces. In addition to PEG, PVP40 was also reported as a good additive for dynamic passivation of silicon dioxide-glass chips, and was even more active than PEG8000. ${ }^{8,9}$

So far, in all the documented dynamic passivation experiments, polymers were mixed with PCR solution prior to loading the reaction mixture into chips. ${ }^{8,18}$ Native PDMS or glass surfaces in aqueous solution are negatively charged, while PVP is positively charged, hence if the surface adsorbed PVP prior to loading the PCR mixture, this may facilitate preventing polymerase being adsorbed onto the surface. So we hypothesized that pre-passivation (forcing the surface to adsorb PVP prior to loading the PCR mixture) may give better performance than in situ passivation (adding the polymer to the PCR mixture, resulting in competitive adsorption with DNA and polymerase during PCR).

Figure 4 shows a comparison of the effects of polymers on PDMS-glass chip PCR using the two dynamic passivation approaches, in situ passivation and pre-passivation.

Figure 4 also shows that when PVP 10000 or PVP 55000 solutions were introduced into the PCR chambers before injecting the PCR mixture, 


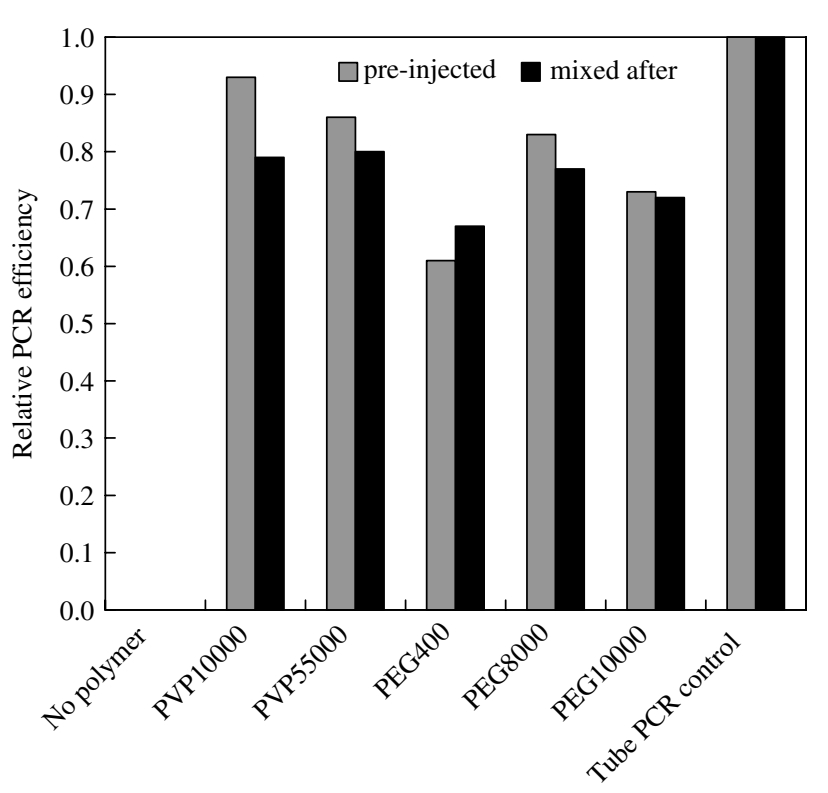

Figure 4. Comparison of in situ passivation and pre-passivation on PDMS-glass chip surface. Pre-passivation (gray bars): polymer solutions were not mixed in PCR mixtures but injected into chip chambers and drained before loading PCR mixtures; In situ passivation (solid bars): polymer solutions were mixed in PCR mixtures; Control: no polymer added to the chip or PCR mixture.

the surface effect of PDMS and glass material on PCR was almost completely erased. The efficiency of passivation was found to follow the order: PVP10000 > PVP55000, PEG8000 > PEG10000 > PEG400. For in situ dynamic passivation, the order of passivation efficiency of PEG and PVP was almost the same as that in pre-PCR passivation: PVP10000, PVP55000 > PEG8000 > PEG10000 > PEG400. There were no PCR products obtained from chip PCR without polymer passivation.

In pre-PCR passivation, the polymers were possibly trapped by the porous PDMS surface and the glass surface. In the presence of PVP, either glass or PDMS adsorbed PVP due to charge-charge interaction. This may be the reason that PVP performed better in chip PCR.

With PEG 400, an opposite effect on passivation efficiency was observed. This may be due to its smaller molecular weight, which would allow it to be released from the surface and diffuse into the solution.

For microchip reactors with large surface-to-volume ratio and material like glass or PDM, considering all the results from tube PCR and chip PCR, the fact that pre-passivation has higher passivation efficiency indicates that competitive adsorption is the principal activity, while the effect of polymers on polymerase is a minor factor during PCR after passivation.

\section{CONCLUSIONS}

Efficient dynamic passivation of PDMS-glass PCR microchip can be achieved using PEG or PVP polymers. Both PEG and PVP have a weak effect on Taq polymerase. Dynamic passivation by pre-injecting polymers into the PDMS-glass microchip provided better results than in situ mixing polymers with PCR reagents. Final polymer concentrations of $0.025 \%$ and $0.4 \%(\mathrm{w} / \mathrm{v})$ in PCR mixtures are efficient. After prepassivation with PVP10000, PVP55000 and PEG8000 solution, the PCR efficiency can recover to $93 \%, 86 \%$ and $83 \%$ respectively, compared to that obtained from tube PCR without the addition of polymer. PCR conducted in PDMS-glass microchip without passivation produced no product. The efficiency of pre-passivation was found to follow the order: PVP10000 > PVP55000, PEG8000 > PEG10000 > PEG400. Competitive adsorption is the principal activity, while the effect of polymers on polymerase plays a secondary role during PCR after passivation.

\section{ACKNOWLEDGEMENTS}

Financial supports from NIH, Michigan Life Sciences Corridor and Program for New Century Excellent Talents in University by Ministry of Education of China are gratefully acknowledged. We thank $\mathrm{Mr}$ Woong Hee Lee and Mr Yusuf Esmail Murgha for their help.

\section{REFERENCES}

1 Yu X, Zhang D, Li T, Hao L and Li X, 3-D microarrays biochip for DNA amplification in polydimethylsiloxane (PDMS) elastomer. Sensors Actuators A: Physical 108:103-107 (2003).

2 Hong JW, Fujii T, Seki M, Yamamoto T and Endo I, Integration of gene amplification and capillary gel electrophoresis on a polydimethylsiloxane-glass hybrid microchip. Electrophoresis 22:328-333 (2001).

3 Hong JW, Fujii T, Seki M, Yamamoto T and Endo I, PDMS (polydimethylsiloxane)-glass hybrid microchip for gene amplification, in 1st Annual International IEEE-EMBS Special Topic Conference on Microtechnologies in Medicine and Biology Proceedings IEEE, Piscataway NJ, ed. by A Dittmar and D Beebe, pp. 407-410 (2000).

4 Martin RS, Gawron AJ and Lunte SM, Dual-electrode electrochemical detection for poly (dimethylsiloxane)-fabricated capillary electrophoresis microchips. Anal Chem 72:3196-3202 (2000).

$5 \mathrm{Xu} Z \mathrm{KK}$, Nie FQ, Qu C, Wan LS and Yao K, Tethering poly (ethylene glycol) $\mathrm{s}$ to improve the surface biocompatibility of poly (acrylonitrile-co-maleic acid) asymmetric membranes. Biomaterials 26:589-598 (2005).

6 Kricka LJ and Wilding P, Microchip PCR. Anal Bioanal Chem 377:820-825 (2003).

7 Giordano BC, Copeland ER and Landers JP, Towards dynamic coating of glass microchip chambers for amplifying DNA via the polymerase chain reaction. Electrophoresis 22:334-340 (2001).

8 Lou XJ, Panaro NJ, Wilding P, Fortina P and Kricka LJ, Increased amplification efficiency of microchip-based PCR by dynamic surface passivation. BioTechniques 36:248-252 (2004).

9 Yang J, Liu Y, Rauch CB, Stevens RL, Liu RH, Lenigk R, et al, High sensitivity PCR assay in plastic micro reactors. Lab Chip 2:179-187 (2002).

10 Fukuba T, Yamamoto T, Naganuma $\mathrm{T}$ and Fujii T, Microfabricated flow-through device for DNA amplification - towards in situ gene analysis. Chem Eng f 101:151-156 (2004).

11 Bickley J and Hopkins D, Inhibitors and enhancers of PCR in Analytical Molecular Biology - Quality and Validation, ed. by 
Saunders G and Parkes H. Redwood Books Ltd, Trowbridge, UK, 81-102 (1999).

12 Maruyama T, Yamamura H, Kotani T, Kamiya N and Goto M, Poly (ethylene glycol)-lipase complexes that are highly active and enantioselective in ionic liquids. Org Biomol Chem 2:1239-1244 (2004).

13 Kingshott P, Thissen $\mathrm{H}$ and Griesser HJ, Effects of cloud-point grafting, chain length, and density of layers on competitive adsorption of ocular proteins. Biomaterials 23:2043-2056 (2002).

14 Kingshott P, McArthur S, Thissen H, Castner DG and Griesser HJ, Ultrasensitive probing of the protein resistance of PEG surfaces by secondary ion mass spectrometry. Biomaterials 23:4775-4785 (2002).
15 Farruggia B, Nerli B, Di Nucci H, Rigatusso R and Picó G, Thermal features of the bovine serum albumin unfolding by polyethylene glycols. Int F Biol Macromol 26:23-33 (1999).

16 Farruggia B, Nerli B and Picó G, Study of the serum albumin polyethyleneglycol interaction to predict the protein partitioning in aqueous two-phase systems. F Chromatogr B 798:25-33 (2003).

17 Irvine DJ, Mayes AM, Satija SK, Barker JG, Sofia-Allgor SJ and Griffith LG, Comparison of tethered star and linear poly (ethylene oxide) for control of biomaterials surface properties. 7 Biomed Mater Res 40:498-503 (1998).

18 Panaro NJ, Lou XJ, Fortina P, Kricka LJ and Wilding P, Surface effects on PCR reactions in Multichip Microfluidic Platforms. Biomed Microdevices 6:75-80 (2004). 